Submitted to the 6th National Conference on Synchrotron Radiation Instrumentation, Berkeley, CA, August 7-10, 1989.

\title{
AN ULTRA-HIGH-VACUUM MULTIPLE GRATING CHAMBER AND SCAN DRIVE WITH IMPROVED GRATING CHANGE
}

\author{
Steven L. Hulbert,
}

NSLS, Brookhaven National Laboratory, Upton, NY 11973

D. J. Holly, F. H. Middleton,

Physical Science Laboratory, University of Wisconsin, Stoughton, WI 53589

and D. J. Wallace

Synchrotron Radiation Center, University of Wisconsin, Stoughton, WI 53589

A new grating chamber and scan drive has been built for the new high flux, high resolution spectroscopy branch line of the TOK hybrid wiggler/undulator on the NSLS VUV ring. The chamber will be used in a Spherical Grating Monochromator (SGM) conniguration.

The grating chamber houses five $180 \mathrm{~mm} \times 35 \mathrm{~mm} \times 30 \mathrm{~mm}$ gratings capable of scanning a range of $12^{\circ}\left(-4^{\circ}\right.$ to $+8^{\circ}$ with respect to the incoming beam direction) for VUV and soft X-ray diffraction.

Gratings can be switched and properly indexed while under vacuum at any scan angle and are mechanically isolated from the vacuum chamber to prevent inaccuracies due to chamber distortions. Gratings can separately be adjusted for height, yaw, pitch, and roll, with the latter three performed while in vacua. The graping chamber has been assembled and tested showing a vacuum of $4.8 \times 10^{-1}$ Torr after being baked to $150^{\circ} \mathrm{C}$. The scan drive provides a resolution of 0.03 arcsec with linearity over the $12^{\circ}$ range of -1.5 arc $\sec$ and absolute reproducibility of 1 arcsec.

The grating chamber was designed and built for the NSLS at Brookhaven National Laboratory by the Physical Sciences Laboratory of the University of Wisconsin. This work was supported by the Division of Material Sciences U. S. Department of Energy under Contract No. DEACO2-76CH00016. 
Presented at the SRI'89 Conference

Berkeley, CA, 8/07-i1/89

\title{
AN ULTRA-HIGH-VACUUM \\ MULTIPLE GRATING CHAMBER \& SCAN DRIVE WITH IMPROVED GRATING CHANGE
}

\author{
Steven L. Hulbert, \\ NSLS, Brookhaven National Laboratory, Upton, NY 11973 \\ Donald J. Holly, Frederic H. Middleton, \\ Physical Sciences Laboratory, University of Wisconsin, Stoughton, WI 53589 \\ and Daniel J. Wallace \\ Synchrotron Radiation Center, University of Wisconsin, Stoughton, WI 53589
}

\begin{abstract}
We describe a new grating chamber and scan drive which has been designed, built, and tested by Physical Sciences Laboratory of the University of Wisconsin for the new high flux, high resolution spectroscopy branch line of the TOK hybrid wiggler/undulator on the NSLS VUV ring. The chamber will contain spherical gratings to be used in the Spherical Grating Monochromator (SGM) configuration introduced by Chen and Sette [1].

The grating chamber houses five $180 \mathrm{~mm} \times 35 \mathrm{~mm} \times 30 \mathrm{~mm}$ gratings capable of scanning a range of $12^{\circ}\left(-4^{\circ}\right.$ to $+8^{\circ}$ with respect to the incoming beam direction) for VUV and soft $X$-ray diffraction.

The gratings can be switched and precisely indexed while under ult:a-high vacuum (UHV) at any sean angle and are mechanically isolated from the vacuum chamber to prevent inaccuracies due to chamber distortions. The gratings, can parately bejadjusted for height, yaw, pitch, and roll, with the latter three performed while in vacuo. The scan drive provides a resolution


of 0.03 are sec with linearity over the $12^{\circ}$ range of $\approx 1.5$ arc sec and absolute reproducibility of 1 arc sec.

This work was supported by the Division of Material Sciences U.S. Department of Energy under Contract No. DE-AC02-76CH00016.

\section{Introduction}

The U13 transverse optical klystron (TOK) wiggler/undulator insertion devices installed on the VUV storage ring at the NSLS is a high flux (22 period) device with a maximum useful flux of $8 \times 10^{15}$ photons $/ \mathrm{sec} / \mathrm{Amp} / 0.1 \% \mathrm{~b} . \mathrm{w}$. when operated in riggler mode $\left(B_{0}=0.86 \mathrm{~T}, \mathrm{~K}=8\right)$. The major overall design criterion for the high flux, high resolution spectroscopy branch line (U13U) on this device is to operate at very high photon energy resolution while maximizing the flux throughput. The optical design layout is that of the Spherical Grating Monochromator $(S G M)^{1}$, in which a Kirkpatrick-Baez front end (a horizontal and vertical collecting and focusing mirror pair) is followed by a monochromator consisting of an entrance slit, a spherical grating, and a movable exit slit.

The grating is the heart of the entire beamline and must be held with great stability while allowing the precise and accurate scan angle rotation which selects a particular photon energy. Since each grating covers only approximately a factor of three in photon energy, a set of five gratings with different groove densities and/or radii is used to cover the entire photon energy sange between $20 \mathrm{eV}$ and $1200 \mathrm{eV}$. Thus, the grating chamber must hold five gratings, each with independent position and angle adjustments (all but one of which must be able to be actuated from outside the evacuated chamber). The carriage which supports the five grating holders must be very rigid and stable, and allow both the extremely precise scan angle rotation and a precise linear grating selection motion.

All of these motions must also meet rigorous reproducibility specifications, since the very high energy resolution spectra that are to be measured with this instrument need to be repeated on 
relatively short time scales in order to achieve good counting statistics and on long time scales to compare one sample with another.

\section{Mechanical Description}

\subsection{General Description}

The grating chamber (Figure 1) has five separate fully adjustable grating assemblies mounted on an across-the-beamline inear indexing carriage that can be indexed while at any scan angle. The carriage is mounted in a rigid, fully counterbalanced underslung scan housing mounted in duplex pairs of extra large angular contact bearings. The bearings fit in pedestals that are independently supported by leg supports with bellows feedthroughs to kinematic mounts on the main frame. The kinematic mounts are for adjustment of the scan housing with respect to the racuum chamber and main frame.

The scan housing has a sine bar drive using an AFBMA grade 3 (spherical within $3 \mu$ inch $(0.08 \mu \mathrm{m})) 440 \mathrm{C}$ stainless steel ball against a chrome carbide drive flat that is flat and parallel within $1 \mu$ in $(0.0254 \mu \mathrm{m})$. For wear, the ball and the flat can easily be repositioned to present. new wear surfaces without requiring recalibration. The flat is adjustable for pitch by means of rotating its housing reiative to the linear actuator drive.

The sine bar drive is an Anorad ${ }^{[2]}$ linear slide having a 10 pitch ball screw driven by a 10,000

microstep per revolution Berger-Lahr ${ }^{(3)}$ five phase stepping motor with a $4: 1$ speed reducer that gives linear positioning resolution of $0.0635 \mu \mathrm{m}$ and $0.03125 \mathrm{arcsec}$ scan resolution per microstep. Position is read by a Heidenhain [4] linear encoder with $0.0781 \mu \mathrm{m}$ resolution by use of a 50 lines $/ \mathrm{mm}$ scale and $256 \mathrm{X}$ logic multiplier.

The grating chamber is rigidly fastened to the frame which is supported by kinematic mounts on the floor. (Figure 2) 


\subsection{Grating Mounts and Adjustment}

Each grating assembly (Figure 3) consists of a base plate, subplate, grating, grating support and hold down means, and grating adjustment mechanism for $X, Y, Z$, roll, pitch, and yaw. It mounts on the linear indexing carriage using a three ball kinematic mount having quarter turn bayonet locking means.

The $\mathrm{Z}$ adjustment is made while the chamber is up to air and the grating assembly is centered on the beamline. It consists of a very fine thread ball-ended screw that supports the grating subplate by means of a subplate ball detent located directly below the grating pole.

$\mathrm{X}$ and $\mathrm{Y}$ adjustments are made by sliding the grating upon fixed three point support pins usirg adjusting screw-spring movements.

Roll, pitch, and yaw adjustments are made using a compound flexural hinge adjuster mechanism that pivots the grating and subplate about the suspension ball used for the $Z$ adjuster. Each has a coarse, medium, and fine adjustment screw that gives the range and resolution required for the installation. In the case of the roll adjustment, resolutions are $2.05^{\circ}, 0.398^{\circ}$, and 72 arc sec per revolution of the respective adjustment screws. For the required resolution of 0.5 are sec, the fine adjustment screw is turned $2.25^{\circ}$. Each of the other adjustments for pitch and yaw has the same fine resolution of $2.25^{\circ}$ per 0.5 arc sec. All three fine adjustments for roll, yaw, and pitch and the pitch and yaw medium adjustments can be made while under vacuum.

\subsection{In-Vacuum Adjustments}

All of the in-vacuum adjustments are made by means of a single bellows-connected rotary-lincargimballed feedthrough (Figure 4) having a hexagonal ball driver to engage the adjustment screws. The adjuster feedthrough is designed with limited linear and gimballed motions to prevent inadvertent contact with grating surfaces. Consequently, it is necessary to make adjustments with the adjustment end of the grating in an elevated $\left(-4^{\circ}\right)$ scan position. 


\subsection{Grating Indexing}

The grating assemblies are mount ed on the top plate of a linear indexing carriage consisting of the top plate, a rectangular tube backbone, and " $\mathrm{V}$ " track that rides between two pairs of adjustable zero-play "V" rollers.

The carriage can be positioned from outside the vacuum by a hand cranked mechanical counter with timing belt speed reduction to a standard hermetically sealed UHV rotary feedthrough with the speed ratio selected that gives 20 turns from one index to the next and a least count of 0.2 for a divisional count resolution of $0.054 \mathrm{~mm}$.

The positioning mechanism is a precision stainless steel lead screw, driven by the vacuum feedthroughs which engages ball-bearing-mounted spring-loaded non-galling stainless steel "V" rollers that provide a rolling contact "nut" mounted on the carriage.

The carriage can be positioned while in any scan position since the threaded roller "nut" is mounted on a ball-bearing-pivoted pendulum whose axis of rotation is on the scan axis. Thus, scan housing rotations are not translated to the nut that positions the carriage because it stays in the same angular position as the drive screw.

\section{Test and Calibration Results}

The monochromator scan drive was calibrated using a laser interferometer with an angular resolution of 0.1 arc sec. If the drive flat is properly adjusted, the Anorad table position indicated on the Heidenhain encoder should ideally be proportional to the sine of the scan angle measured by the interferometer. $[5]$

Figure 5 shows the deviation from sinusoidal drive measured with the laser interferometer. Note that the drive is truly sinusoidal to within approximately $\pm 1.5 \mathrm{arc} \mathrm{sec}$, and that the scan angle is repeatable to about 1 are sec. Other measurements suggest that the abrupt excursion seen at small positive angles is associated with an irregularity in the scan pivot ball bearings. 
An autocollimator with a resolution of 0.2 arc sec was used to measure angle adjustment range and resolution. The autocollimator was also used to measure the grating angle changes caused by removing and replacing the grating mount assemblies from the grating table.

Calibration tests are summarized as follows:

1. Scan is reproducible to 1 arc sec and corresponds to a true sine drive with \pm 1.5 arc sec.

2. Linear indexing positioning accuracy is within $\pm 0.005 \mathrm{~mm}$ at different scan angles.

3. Linear indexing results in:

(A) Individual grating $\mathrm{Z}$ variations of not more than $\pm 0.0004 \mathrm{~mm}$ at different scan angles.

(B) Individual grating pitch variations of \pm 3.7 are sec.

(C) Individual grating roll variations of \pm 4.2 arc sec.

(D) Individual grating yaw variations of $\pm 7.8 \mathrm{arc} \mathrm{sec}$.

4. Grating removal - replacement results in:

(A) A verage yaw variation of 2.6 arc sec

(B) A verage pitch variation of 6.3 arc sec.

(C) A verage roll variation of 17.8 arc sec.

5. $Z$ adjustment range of $\pm 3 \mathrm{num}$ with resolution of $\pm 0.001 \mathrm{~m}$.

6. Yaw fine adjustment range of \pm 0.05 degrees using fine and medium adjustment screws and resolution of \pm 0.2 arc sec.

7. Roll fine adjustment range of \pm 0.05 degrees and resolution better than 0.5 arc sec.

8. Pitch fine adjustment range of \pm 0.09 degrees and resolution of 0.5 arc sec. 
Vacuum tests included an RGA test before, during, and after a $150^{\circ} \mathrm{C}$ extended bakeout. There was no significant contamination and virtually nothing with a molecular weight greater than 44. Continuous pumping with a $220 \mathrm{l} / \mathrm{s}$ ion pump and intermittent firing of a Ti sublimator resulted in a final pressure of less than $5 \times 10^{-11}$ Torr.

\section{Conclusions}

The linear indexing multiple grating chamber and scan drive provide precise and reproducible grating scan and linear indexing. The scan is very nearly truly sinusoidal. The chamber is UHV. compatiole, and grating adjustments are easily performed in vacuo.

\section{References}

[1] C. T. Chen and F. Sette, Nucl. Instrum. Meth. A256, 595 (1987).

[2] Anorad Corporation, 110 Oser Avenue; Haupfauge, New York; (516) 231-1990

[3] Berger-Lahr Corporation, 1-T Fitzgerald Drive; Jeffrey, New Hampshire, 03452; (603) 532-7701

[4] Heidenhain Corporation, 80 North Scott Street; Elk Grove Village, Illinois; (312)593-6161

[5] Daniel J. Wallace, Roger W.C. Hanson, F. Keith Perkins, Charles H. Pruett, John Welnak,

"Alignment Techniques for Calibration and Installation of a 6M Toroidal Grating Monochromator, " published in this Proceedings.

\section{Figure Captions}

1. Scan housing and support, counter balance, sine bar drive, linear indexing carriage, indexing pendulum drive, and grating assemblies.

2. Grating chamber assembly with kinematic mounts for scan assembly and frame.

3. Grating pitch, roll, yaw and $z$ adjustment mechanism. 


\title{
4. Linear-rotary-gimballed feedthrough with ball adjuster for grating adjustments.
}

5. Deviation from true sinusoidal drive. Laser interferometer data from five scans are overlaid to show repeatability.

$f i l \theta=d 3\left[a d m i n \_j a b\right.$. middleton] 8 siBg_2.tex

\section{DISCLAIMER}

\begin{abstract}
This report was prepared as an account of work sponsored by an agency of the United States Government. Neither the United States Government nor any agency thereof, nor any of their employees, makes any warranty, express or implied, or assumes any legal liability or responsibility for the accuracy, completeness, or usefulness of any information, apparatus, product, or process disclosed, or represents that its use would not infringe privately owned rights. Reference herein to any specific commercial prodict, process, or service by trade name, trademark, manufacturer, or otherwise does not necessarily constitute or imply its endorsement, recommendation, or favoring by the United States Government or any agency thereof. The views and opinions of authors expressed herein do not necessarily state or reflect those of the United States Government or any agency thereof.
\end{abstract}



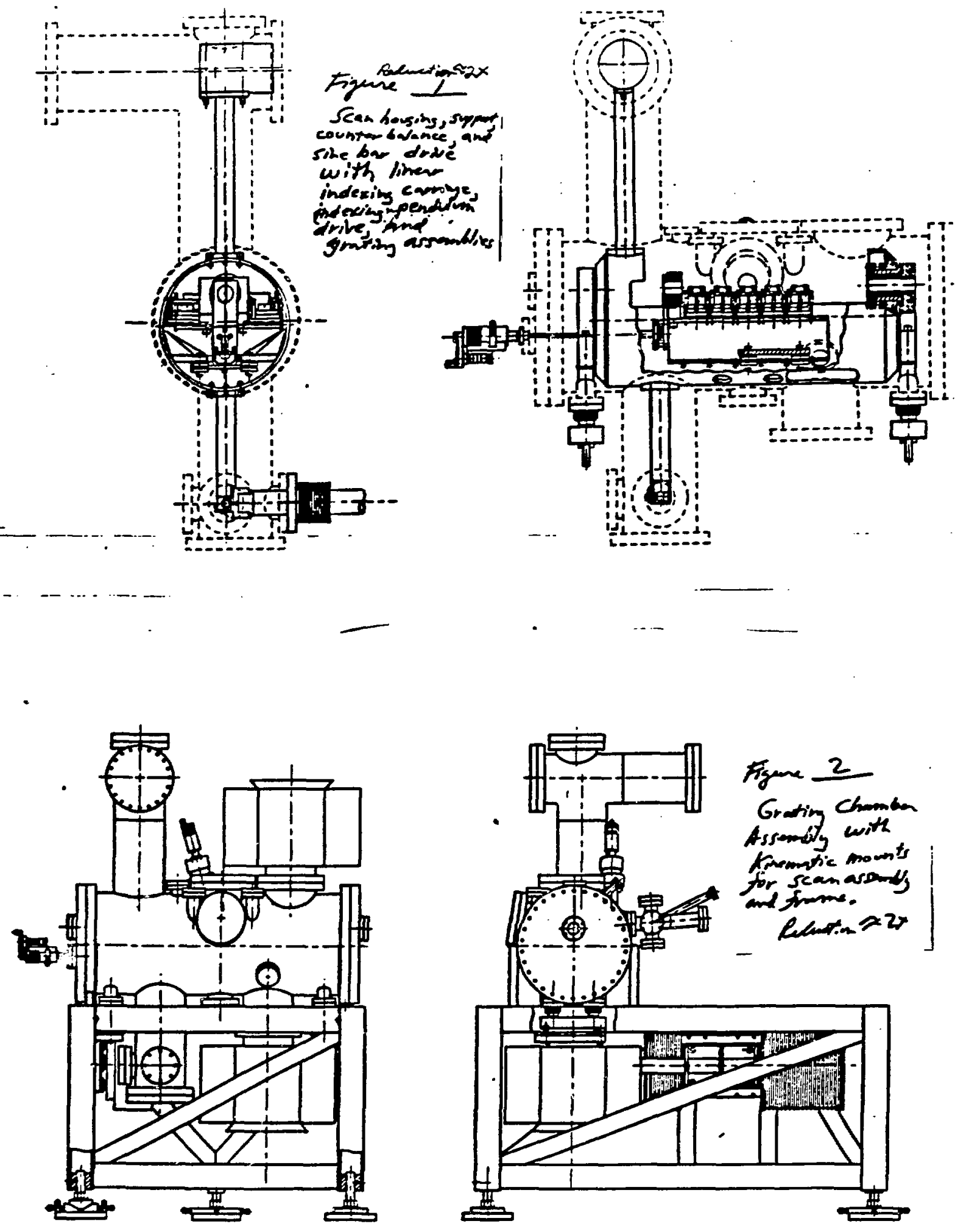

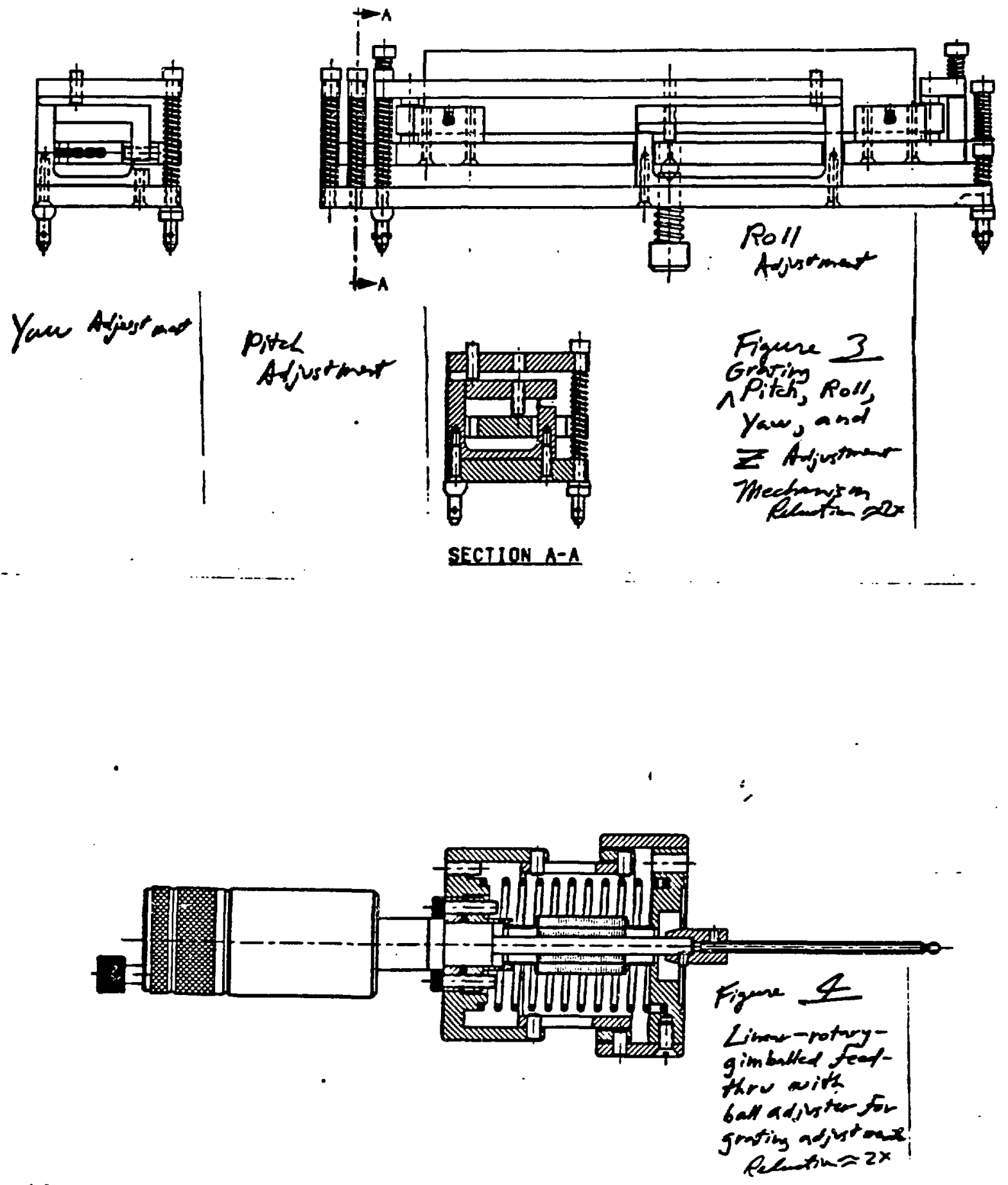


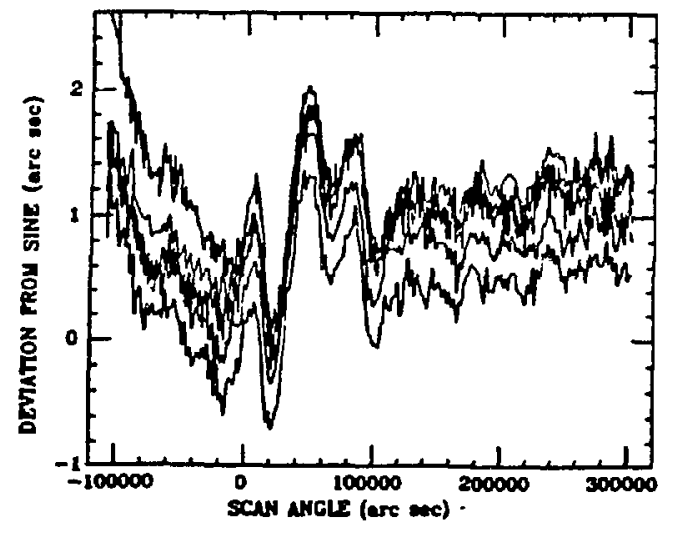

FIG. 5. DEVIATION FROM TRUE SINUSOIDAL DRIVE. LASER INTERFET OMITTR DKA FROIA FIVE SCANS ARF OVEKLAID TO SHOW REPEAT ABILITY. 